\title{
Germanica
}

\section{Celans „rumänische Büffel“}

Eine Czernowitzer Reminiszenz

«Les bison

$s$ roumains " de Paul Celan. Une réminiscence de Czernowitz

Paul Celan's "Romanian Buffaloes". Reminiscence from Czernowitz

\section{Andrei Corbea-Hoisie}

\section{(2) OpenEdition}

\section{Journals}

Édition électronique

URL : http://journals.openedition.org/germanica/4545

DOI : 10.4000/germanica.4545

ISSN : 2107-0784

Éditeur

Université de Lille

Édition imprimée

Date de publication : 31 juillet 2018

Pagination : 77-96

ISBN : 9782913857414

ISSN : 0984-2632

\section{Référence électronique}

Andrei Corbea-Hoisie, „Celans „rumänische Büffel““, Germanica [Online], 62 | 2018, Online erschienen am: 31 Juli 2020, abgerufen am 14 Januar 2021. URL: http://journals.openedition.org/germanica/ 4545 ; DOI: https://doi.org/10.4000/germanica.4545 


\title{
Celans „,rumänische Büffel““. Eine Czernowitzer Reminiszenz
}

\author{
Andrei CORBEA-HoISIE \\ Universität Iaşi
}

\author{
Auch deine \\ Wunde, Rosa. \\ Und das Hörnerlicht deiner \\ rumänischen Büffel \\ an Sternes Statt überm \\ Sandbett, im \\ Redenden, rot- \\ Aschengewaltigen \\ Kolben.
}

Paul Celans Gedicht Coagula, das zum 1967 veröffentlichten Band Atemwende gehört ${ }^{1}$, ist laut den vom Dichter selbst vorgenommenen Datierungen am 20. Februar 1965 entstanden, und zwar am selben Tag wie das Gedicht Solve, mit dem es als Paar-bezogen auf die alchemische Formel „,solve et coagula“ („Löse und verbinde“) - konzipiert wurde ${ }^{2}$. Es

1. - Paul Celan, Werke. Historisch-kritische Ausgabe besorgt von der Bonner Arbeitsstelle für die Celan-Ausgabe, hrsg. v. Beda Allemann, Rolf Bücher, Axel Gellhaus, Stefan Reichert [weiter unter der Sigle BA], hier Bd. 7,1, Atemwende, hrsg. v. Rolf Bücher, Frankfurt a.M., Suhrkamp, 1990, S. 83; Paul Celan, Werke. Tübinger Ausgabe, hrsg. v. Jürgen Wertheimer [weiter unter der Sigle TA], hier Atemwende, Vorstufe-Textgenese-Endfassung, bearbeitet v. Heino Schmull u. Christiane Wittkopp, Frankfurt a.M., Suhrkamp, 2000, S. 139.

2. - Vgl. BA, Bd. 7,2, hrsg. v. Rolf Bücher, S. 188; TA, S. 138. 
wird in der Exegese allgemein festgehalten, dass erste Entwürfe aus dem Jahr 1962 im Verhältnis zu dem Vorhaben des Dichters stammen, eine Walliser bzw. Pariser Elegie zu schreiben, die den Niemandsrose-Band abschließen sollte ${ }^{3}$, während die Reflexion über das mit dem dichterischen Schaffen symbolisch verbundene Titelpaar schon in den Notizen für die Meridian-Rede dokumentiert wird ${ }^{4}$. Die textbezogenen CelanKommentare haben offensichtlich diese ,doppelte Interpretationslinie bevorzugt: ein Aufsatz Anders Ollsons steht als hervorragendes - weil keinesfalls dogmatisches - Modell für diese Sichtweise ${ }^{5}$.

Für Wolfgang Emmerich eignete sich dasselbe Gedicht durchaus, jenes poetische Verfahren zu veranschaulichen, das den ,rätselhaften, nur ,entfernt ${ }^{\star}$ verständlichen Text, [...] von dem der Leser in der Tat immer wieder den Eindruck haben kann, dass er auf einer anderen Raum- und Zeitebene angesiedelt ist als er selbst", aus allerlei konkreten Bezügen auf die Lebenswelt und -geschichte des Dichters konstituiert6; Celan selbst, der sonst fast nie seine Gedichte zu ,erklären' neigte, versuchte in einem Brief an den Bukarester Freund Petre Solomon gerade aufgrund des Coagula-Textes diesen Schaffensprozess zu beschreiben: „les bisons roumains aperçus par Rosa Luxembourg à travers les barreaux de sa prison convergent avec les trois mots du Médecin de campagne de Kafka - et avec ce nom: Rosa. Je coagule, j'essaie de faire coaguler [...]"7. Dadurch wird auch die stets wiederholte Behauptung Celans einsichtig, seine Gedichte seien zwar ,eine Erscheinungsform der Sprache“, aber „,kein Zeichen-System“, sondern sie kämen ,zur Welt [...] mit Welt befrachtet zu ihr, der Welt" ${ }^{4}$. Auf

3. - Vgl. u.a. Axel Gellhaus, ,Wortlandschaften. Konzeption und Textprozesse bei Celan“, in: Axel Gellhaus, Karin Herrmann (Hg.), „Qualitativer Wechsel“. Textgenese bei Paul Celan, Würzburg, Königshausen \& Neumann, 2010, S. 11-68; Jörg Therstappen, „Das Projekt einer ,Pariser Elegie““, in: Albrecht Rieder, Jörg Therstappen (Hg.), „Opferstatt meiner Hände“. Die Paris-Gedichte Paul Celans, Würzburg, Königshausen \& Neumann, 2017, S. 111-118.

4. - Vgl. TA, Der Meridian. Endfassung, Vorstufen, Materialien, hrsg. v. Bernhard Böschenstein u. Heino Schmull u. Mitarbeit von Michael Schwarzkopf und Christiane Wittkopp, Frankfurt a.M., Suhrkamp, 1999, S. 65, 230. Celan habe die Anregung aus Hofmannsthals Erzählung Andreas oder die Vereinigten übernommen. Vgl. auch Bernhard Böschenstein, „Celan lecteur des Notes de Hofmannsthal“, Austriaca, Jg. 18, Nr. 37 (Dez. 1993), S. 49-60, hier 49, 59.

5. - Anders Olsson, „Spectral Analysis. A Commentary on ,Solve“ and ,Coagula““, in: Aris Fioretos (Hg.), Traces. Readings of Paul Celan, Baltimore u. London, John Hopkins University Press, 1994, S. 267-279.

6. - Wolfgang Emmerich, Paul Celan, Reinbek bei Hamburg, Rowohlt, 1999, S. $14 \mathrm{ff}$.

7. - Paul Celan an Petre Solomon in dem Brief vom 23. XI. 1967, in: Petre Solomon, Dimensiunea românească, București, Kriterion, 1987, S. 237-239, hier 238.

8. - Paul Celan, Mikrolythen sinds, Steinchen.Die Prosa aus dem Nachlass, hrsg. v. Barbara Wiedemann u. Bertrand Badiou, Frankfurt a.M., Suhrkamp, 2005, S. 138 ff. 
Behauptungen wie jene Günter Blöckers aus der berüchtigten Rezension zum Sprachgitter-Band in Der Tagesspiegel vom 11. Oktober 1959, laut der „Celans Verse [...] vorwiegend graphische Gebilde [sind]“, wusste der Dichter zu antworten, indem er immer wieder betonte, das Gedicht werde dem Leser allein von dem „Atem dessen, der - sterblich - durch das Gedicht geht" wird der poetische Text zu einem ,auf die Kreatur zu beziehende[n] Wort" 11 desjenigen, der ,unter dem Neigungswinkel seines Daseins [...] spricht"12. Damit bedeutete für ihn sein Ruf ,Je suis la poésie!"13 keinen leeren Leitsatz - den Peter Szondi genau begriffen hatte, als er sich vornahm, zu prüfen, inwiefern das von Celan in Berlin im Dezember 1967 geschriebene Gedicht Du liegst „durch ihm Äußerliches bedingt“ wurde, auch wenn letztendlich „,solche Fremdbestimmung [...] durch die eigene Logik des Gedichts" aufgehoben werden sollte ${ }^{14}$. Mit der denkwürdigen Formel der „Unendlichkeitssprechung von lauter Sterblichkeit" 15 nannte Celan selbst diese spezifische Dialektik der Dichtung als ,Gerinnen' von Anlässen und Vorkommnissen in ein sinngebend gesprochenes Gedenken.

9. - Ebd., S. 143.

10. - TA, Der Meridian, a.a.O., S. 4.

11. - Ebd., S. 8.

12. - Ebd., S. 9.

13. - Jean Bollack, Herzstein. Über ein unveröffentliches Gedicht von Paul Celan, München, Carl Hanser [Edition Akzente], 1993, S. 11.

14. - Vgl. Peter Szondi, „Eden“, in: Ders., Celan-Studien, Frankfurt a.M., Suhrkamp, 1972, S. 120. Als Replik auf Szondis Standpunkt hat Hans Georg Gadamer dasselbe Gedicht ,textimmanent“ interpretiert und gemeint, dass man „nichts Privates und Ephemeres wissen [muss]. Man muss sogar, wenn man es weiß, wegdenken und nur das denken, was das Gedicht weiß“. Vgl. Hans-Georg Gadamer, Wer bin ich und wer bist du? Kommentar zu Celans „Atemkristall“, Frankfurt a.M., Suhrkamp, 1986 [revidierte und ergänzte Ausgabe], S. 125-130. Vgl. auch Jean Bollacks radikale Kritik der Position Gadamers in Jean Bollack, Paul Celan. Poetik der Fremdheit, Wien, Zsolnay, 2000. Dazu noch Jürg Berthold, „,Wir müssen's wohl leiden'. Formen ,autobiographischen“ Schreibens“, Poetica, Jg. 24 (1992), S. 90-101; Thomas Schröder, „Undeutbarkeit. Annäherungen an Peter Szondi anlässlich seiner Fragment gebliebenen Celan-Interpretation Eden, Berlin“, in: Richard Faber, Eva-Maria Ziege (Hg.), Das Feld der Frankfurter Kultur- und Sozialwissenschaften nach 1945, Würzburg, Königshausen \& Neumann, 2008, S. 123-138; Martin Schierbaum, ,,Aber das Gedicht spricht ja'. Ethik und Textualität in Celans Gedichten Schibboleth und Du liegst", in: Steffi Hobuß, Nicola Tams (Hg.), Lassen und Tun. Kulturphilosophische Debatten zum Verhältnis von Gabe und kulturellen Praktiken, Bielefeld, Transcript Verlag, 2014, S. 119-158.

15. - TA, Der Meridian, a.a.O., S. 11. Vgl. auch Emmerich, Paul Celan, a.a.O., S. 16. 
In dem alchemischen Wechselspiel von solve und coagula ging es Celan um die zyklische Renaissance der „Form“ aus dem Elementarmagma einer durch „Gift-pfalzen“ zugrundegegangenen Ordnung, deren Auflösung selbst die "Schrift“ miteinbezieht; das rubedo des Lebens lässt sich dennoch im „aschengewaltigen / Kolben“ blicken und (,,redend“) hören, obwohl dies mit dem Leid der blutenden „Wunde“ verbunden sei. Vom Himmel aus wachen auf die nächtlich ruhige Landschaft lichtspendende Büffelhörner: Das Bild wird mit der symbolischen Kraft von Davids Hörnern des Heils (wie im 18. Psalm) und gleichzeitig der Füllhörner Amaltheas aus der griechischen Mythologie evoziert, die die kommende Fruchtbarkeit der in ihren Konturen wieder hergestellten Welt versprechen. Die „Wunde“ Rosas, der „rosa alchemyca“, deren Name laut der esoterischen Tradition des „alchemischen“ Wissens jenen der (Muttergottes) Maria zu verhüllen vermag, kann - auch in Anlehnung an Kafkas vergewaltigte Magd namens Rosa aus dem Landarzt - eine Hoffnung auf den weltrettenden Sohn verkünden ${ }^{16}$.

Diese , ausgeglichene' Interpretation von Coagula wird dennoch von einem Adjektiv ,getrübt‘: Es sind die Hörner ,rumänischer Büffel“, die ihrerseits mit dem Namen Rosa assoziiert werden. Die vermeintlich rätselhafte Zuweisung war ohne allzugroße Mühe zu lösen: Celan berief sich auf eine Sequenz aus einem bekannten und vielfach kommentierten Brief Rosa Luxemburgs aus dem Breslauer Gefängnis im Dezember 1917, in dem sie über eine von ihrer Zelle beobachtete Szene mit gequälten Büffeln berichtete, die angeblich aus Rumänien als Kriegstrophäen hierher gebracht wurden, um bei schweren Lasttransporten benutzt zu werden; auf die Grausamkeit blickend, mit der rohe Soldaten die blutenden Tiere auspeitschten, ließ sie damals ihren Emotionen freien Lauf: „O, mein armer Büffel, mein armer, geliebter Bruder, wir stehen hier beide so ohnmächtig und stumpf und sind nur eins in Schmerz, in Ohnmacht, in Sehnsucht" ${ }^{17}$. Nur zwei Jahre später, im Januar 1919, sollte die „Spartakus"-Führerin während des Berliner Arbeiteraufstandes von Freikorps-Soldaten festgenommen und barbarisch - durch wilde Schläge mit Gewehrkolben - ermordet werden ${ }^{18}$.

Die Frage, die sich dabei zwangsläufig stellen lässt, betrifft erstmal den eventuellen Sinn-Zusammenhang, den Celan durch die Überlagerung des Themenkomplexes um die alchemische Alternanz

16. - Vgl. Olsson, „Spectral Analysis“, a.a.O.

17. - Rosa Luxemburg, Briefe aus dem Gefängnis, Berlin-Schöneberg, Verlag der Jugendinternationale, 1922, S. 60.

18. - Elisabeth Hannover-Drück, Heinrich Hannover (Hg.), Der Mord an Rosa Luxemburg und Karl Liebknecht. Dokumentation eines politischen Verbrechens, Frankfurt a.M., Suhrkamp, 1967. 
solve und coagula mit dem Luxemburg-Zitat berücksichtigt hatte. Oder durfte der Name Rosa den einzigen Impuls zu der von Celan selbst heraufbeschwörten „Konvergenz“ darstellen, die das „Gerinnen“ - ein Wort, das spontan an das Blut der Rosa Luxemburg und ebenso ihrer „rumänischen Büffel“ erinnert - als eine zum Verstehen einladende „Flaschenpost" an die Leser ${ }^{19}$ auslöste? Schon Michael Hamburger hat bei seiner Übersetzungsarbeit des Gedichtes Coagula ins Englische auf die Schwierigkeit einer eindeutigen Interpretation dieser scheinbar gegensätzlichen Bedeutungsstränge hingewiesen, als er die komplizierte Semantik des Lexems „Kolben“ zur Diskussion brachte, dessen Interpretation/Übersetzung die Gewichte der Auslegung in die eine oder in die andere Richtung verrücken konnten ${ }^{20}$. Der Übersetzer hat seine Wahl getroffen, wohl meinend, dass er auf diese Weise der Autorenintention entgegenkommt. Eigentlich mündet aber jeglicher Kommentar auch in diesem Fall in die langwierige hermeneutische Debatte über die Prävalenz von Erfahrungshorizonten der Leser oder der vom Dichter selbst eingebauten Leseanweisungen bei der Interpretation seiner Texte ${ }^{21}$. Die Tatsache, dass ein Peter Horst Neumann, der zu den erfahrensten Lesern des Celanschen Werkes gehörte, seine manchmal schiere Ratlosigkeit vor dessen hermetischen Texten erklärte, bis er dazu kam, sich zu fragen, ,was muss ich wissen, um zu verstehen“22, kann man nur als eine Ermunterung auffassen, den letzteren Weg zu beschreiten, d.h. die Genese des Gedichtes in allen zeitlichen Phasen und Stadien zu begleiten. Gerade diesen Vorgang möchten wir im Folgenden prüfen, um zu versuchen, durch die Projektion des Syntagmas „rumänische Büffel" in den Entstehungsprozess von Coagula näher an die lyrisch gestaltende Gedankenwelt des Dichters zu gelangen.

Wie schon erwähnt, kann die Genese des Gedichtes Coagula im Zeitraum der Ausformung des Lyrikbandes Atemwende von dessen ersten Textversionen, die einen Bestandteil der 1962 datierten Skizzen für einen als Abschluss der Niemandsrose gedachten elegischen Zyklus - in einem gewissen Dialog mit Rilkes Duineser Elegien ${ }^{23}$ - bilden,

19. - „Immerzu nur lesen, das Verständnis kommt von selbst“, soll Celan einmal zu Israel Chalfen gesagt haben, vgl. Israel Chalfen, Paul Celan. Eine Biographie seiner Jugend, Frankfurt a.M., Suhrkamp Taschenbuch, 1983, S. 7.

20. - Vgl. Michael Hamburger, „Celan Übersetzen“, Text+Kritik, H. 53/54, 3. Auflage, Neufassung, München, Edition Text+Kritik, 2002, S. 8-24.

21. - Vgl. Anm. 14. Vgl. auch Andrei Corbea-Hoisie, Paul Celans „unbequemes Zuhause": sein erstes Jahrzehnt in Paris, Aachen, Rimbaud, 2017.

22. - Peter Horst Neumann, Zur Lyrik Paul Celans. Eine Einführung, Göttingen, Vandenhoeck\&Ruprecht, 1990, S. 100.

23. - Vgl. die Anmerkungen von Barbara Wiedemann und Bertrand Badiou zum 
nicht getrennt werden. Die erste Variante aus dem Jahre 1965 übernimmt die letzte aus dem Komplex der Walliser bzw. Pariser Elegie mit allen ihren inhaltlichen Elementen: „Wunde“, „Rosa“, „Hörnerlicht der rumänischen Büffel“, „Sterne“, „Sandbett“, „Kolben“; beibehalten wird ebenso das Adverb ,auch“ als Incipit, wie auch die Formel „deiner - auch meiner - rumänischen Büffel“, die in der Endversion des Gedichtes durch die Streichung des Einschubs „,auch meiner“24 als ,vereinfacht ${ }^{*}$ - inklusive durch eine andere Verseinteilung - erscheint ${ }^{25}$. Es leuchtet schon beim Ansehen der Manuskriptseite ein, dass die ,alchemische“ Thematik die Intentionalität des Autors zu dieser Zeit beherrscht: Er sieht hier ausdrücklich vor, dass Coagula als ,Paar" vom am selben Tag neu entstandenen Solve gedruckt werden soll26. Mit anderen Worten konzentriert sich Celan offensichtlich auf das physischgeistige ,Wunder" des „Gerinnens“ (coaguler); ohne ihre eigene Brisanz zu verbüßen, dürfen die einzelnen zusammengebrachten Bezüge für die Gabe des dichtenden Subjekts zeugen, in der Natur wie auch im Geiste jene Korrespondenzen zwischen unterschiedlichen Elementen zu entdecken, die zu einer neuen Kohärenz führen können.

Der Schritt zurück in die Schaffensperiode der beabsichtigten elegischen Vollendung des Niemandsrose-Bandes ergibt in diesem anderen Kon-Text ein ganz unterschiedliches Bild von Celans Verhältnis zur ,Materie der damaligen Coagula-Version. Die ersten Blätter aus der Sammelmappe mit Texten im Zusammenhang mit der Walliser Elegie halten Daten aus dem Jahr 1961 fest $^{27}$; das Schreibblock-Blatt, auf dem Celan die ursprüngliche Fassung von Coagula noch unter der Überschrift Pariser Elegie notierte, wurde von ihm wieder verwendet, indem er darauf auch den fast definitiven Entwurf für den AtemwendeBand - mit dem Vermerk „1962 $\rightarrow$ 20.II.1965“ - niederschrieb ${ }^{28}$.

Gedicht Walliser Elegie in Paul Celan, Gesammelte Werke in sieben Bänden [weiterhin GW], hrsg. v. Bertrand Badiou, Jean-Claude Rambach u. Barbara Wiedemann, Frankfurt a.M., Suhrkamp, 1993, hier Bd. 7, S. 380. Vgl. auch James K. Lyon, „,Rilke und Celan“, in: Amy D. Colin (Hg.), Argumentum e Silentio. International Paul Celan Symposium, Berlin/New York, de Gruyter, 1987, S. 199-213.

24. - Die Löschung von „Spuren konkreter biographisch-anekdotischer Zusammenhänge“" während des Textprozesses - ein bei Celan gebräuchliches Prozedere - soll laut Axel Gellhaus und Karin Herrmann keinesfalls als „Akt der Hermetisierung“ betrachtet werden, sondern als eine „Durchtrennung der Linearität vom anekdotischen Anlass zur Bedeutung des Gedichts“, vgl. Axel Gellhaus, Karin Herrmann, „Vorwort“, in: Dies., „Qualitativer Wechsel“, a.a.O., S. 7-10, hier 9.

25. - BA, 7,2, S. 188-190; TA [Atemwende], S. 138-139.

26. - Vgl. Faksimile in TA [Atemwende], S. 202.

27. - BA, Bd. 11, hrsg. v. Holger Gehle u. Thomas Schneider unter Mitarbeit von Andreas Lohr in Verbindung mit Rolf Bücher, Frankfurt a.M., Suhrkamp, 2006, S. $393 \mathrm{ff}$.

28. - Ebd., S. 456. Vgl. auch Faksimile in TA [Atemwende], S. 202. 
Die zum Projekt der Elegie gehörenden Manuskriptseiten belegen das Auftauchen von gedanklichen Impulsen, deren unmittelbare Verbindung mit dem endgültigen Text naheliegend ist: Am 18. „Feber“ 1962 wird Kafkas Landarzt ausdrücklich in einem Vermerk erwähnt, der u. a. - auch mit direktem Bezug auf Rilkes berühmte Grabschrift - die Vokabeln „Rose“, „Wunde“, „Rosa“ enthielt ${ }^{29}$; ein Vers aus einem Entwurf von oder nach dem 20. Mai 1962 lautet „Die Wunde. Rosa“ und wird ebenfalls durch einen Verweis auf Kafkas Landarzt fortgesetzt ${ }^{30}$; eine Reihe von ab dem 24. Mai 1962 verfassten Versskizzen führen das Morphem ,auch“ als Gedichtincipit an ${ }^{31}$; am 7. November 1962 notierte Celan mit einem Bleistift auf dasselbe Blatt, auf dem mit Tinte ein Entwurf zur Ergänzung des Gedichtes Eine Gauner- und Ganovenweise festgehalten war, die getrennten Zeilen Die rumän. Büffel // Rosa Luxemburg // - deine Wunde, Rosa - //32 - allerdings hatte er schon auf einen Briefumschlag mit dem Poststempel vom 5. Oktober 1962 die zwei Zeilen Die rumänischen Büffel und (Auch deine Wunde, Rosa) aufgezeichnet ${ }^{33}$. Am 6. Dezember $1962^{34}$ trug er schließlich in einen Schreibblock eine angeblich für die Arbeit an dem NiemandsroseBand bestimmte Notiz ein: Auch deine Wunde, Rosa (R.L. Briefe aus dem G-s wiederlesen, event. auch Ernst Toller Briefe a.d.G-s $)^{35}$.

Die Zeitspanne, über die sich die mühsame Auseinandersetzung mit dem Projekt der Walliser/Pariser Elegie erstreckte, aus dem sich außer Coagula immerhin mehrere Gedichte aus der Niemandsrose ${ }^{36}$

29. - BA. 11, S. 420. Celan hat Kafkas Landarzt gründlich für seinen Literaturunterricht an der École Normale Supérieure im Studienjahr 1959-1960 exzerptiert, vgl. Patrick Difour, „- - wo ich also lesen, wo ich vor-, mit- und nachlesen kann -“. Paul Celans Lehrtätigkeit an den Écoles normales supérieures von Saint-Cloud und der Rue d'Ulm, Dissertation, Paris, 2011, S. 180-190. Zur einschlägigen Rolle des Namens Rosa bei der Namensgebung des Lyrikbandes Niemandsrose vgl. A. Gellhaus, „Wortlandschaften“, a.a.O., S. 31. Über Celans Interesse für die Grabschrift Rilkes vgl. J.K. Lyon, „Rilke und Celan“, a.a.O., S. 205 ff.

30. - BA, 11, S. 434; TA [Atemwende], S. 138.

31. - BA, 11, S. $436 \mathrm{ff}$.

32. - Ebd., S. 454. Vgl. auch BA, Bd. 6,2, hrsg. v. Axel Gellhaus unter Mitarbeit v. Holger Gehle u. Andreas Lohr in Verbindung mit Rolf Bücher, Frankfurt a.M., Suhrkamp, 2001, S. 120.

33. - BA, 7,2, S. 188; BA, 11, S. 455; TA [Atemwende] S. 138.

34. - In TA [Atemwende], S. 138, wird das Datum ,6.12.1962“ angeführt; in der BA, 11, S. 455, wird die Datierung nuanciert: ,wahrscheinlich kurz nach dem 6.12.1962“.

35. - Er meinte die seit 1920 in zahlreichen Editionen erschienene Sammlung der Briefe aus dem Gefängnis von Rosa Luxemburg und ebenfalls Ernst Tollers Briefe aus dem Gefängnis (Amsterdam, Querido, 1935).

36. - Es handelt sich um In Eins, Hinausgekrönt, Hüttenfenster, Es ist alles anders, In der Luft, vgl. TA, Niemandsrose. Vorstufe-Textgenese-Endfassung, bearbeitet v. Heino Schmull unter Mitarbeit v. Michael Schwarzkopf, Frankfurt a.M., Suhrkamp, 1996, S. IX; Coagula wanderte dann in den nächsten Gedichtband Atemwende. 
abnabelten, war alles andere als ruhig in Celans Leben. Die Folgen der Plagiatsvorwürfe lasteten schwer auf seinem psychischen Zustand, auch wenn der Höhepunkt der „Affäre“ 1962 eigentlich überwunden war; was ihn jedoch jetzt quälend beschäftigte, steht ausdrücklich in einem Brief vom 5.8.1962 an Klaus Wagenbach, seinen damaligen Lektor im S. Fischer Verlag: Er habe ,zumindest von einigen: Solidarität" erwartet und war enttäuscht, dass diese nicht in der Art und Weise, die er sich vorgestellt hatte („Entlarvung der Machenschaften“ z. B.), ausgedrückt wurde ${ }^{37}$. Mehrere Briefe, Briefentwürfe und Notizen aus diesen Monaten ${ }^{38}$ zeugen von einem wachsenden Einsamkeitsgefühl, untermalt von einem drückenden Misstrauen gegen allerlei Leute einschließlich gegen enge Freunde wie Klaus Demus ${ }^{39}$. Die manchmal gebrochene Diktion dieser Fragmente dürfte ebenfalls auf eine unstabile Gemütslage des Dichters, auf eine Art beunruhigte Aufregung hindeuten; der Bericht Dürrenmatts über einen überraschenden Besuch Celans bei ihm in Neuchâtel weist auch in diese Richtung ${ }^{40}$. Nicht minder der ,Stoff', aus dem sich die verschiedenen Notate und Konzepte des geplanten elegischen Zyklus nährten, stellt neben dem dichten intertextuellen Netz (von Dante bis zu Rilke und Mandelstam) einen sprunghaften Gedankenfluss dar, der u. a. - mit Rücksicht auf seine eigene Lage - die Ratlosigkeit über das marginale Schicksal des Poeten in einer ihm gegenüber verfeindeten Gesellschaft, das offen behauptete jüdische Bewusstsein des deutschsprachigen Dichters, die radikal kämpferische Disposition anhand alter Revolutionsdaten, -orte und -bilder, und ebenso verzweifelte, extrem behauptete Erotik zusammenknüpft. Am Beispiel der Entstehung des Gedichtes In eins hat Axel Gellhaus am überzeugendsten diesen verzwickten - auf den ersten Blick sogar verworrenen - Weg durch das Motivgeflecht der Elegie bis zum endgültigen Text genau rekonstruiert ${ }^{41}$.

37. - Vgl. Paul Celan - Die Goll-Affäre. Dokumente zu einer »Infamie«, zusammengestellt, hrsg. u. kommentiert v. Barbara Wiedemann, Frankfurt a.M., Suhrkamp, 2000, S. 578.

38. - Ebd. Vgl. auch „Chronologie“, in: Paul Celan, Gisèle Celan-Lestrange, Correspondance, éd. et commentée par Bertrand Badiou avec le concours d'Éric Celan, Paris, Seuil, 2001, Bd. 2, S. 533-541.

39. - Vgl. Paul Celan - Klaus und Nani Demus, Briefwechsel, hrsg. u. komm. v. Joachim Seng, Frankfurt a.M., Suhrkamp, 2009, besonders den Brief Klaus Demus' an Celan vom 17.VI.62, S. 435 f.

40. - Vgl. Friedrich Dürrenmatt, „Erinnerungen an Paul Celan“, in: Ders., Turmbau. Stoffe IV-IX, Zürich, Diogenes, 1990, S. 169-171. Die „Chronologie“ (in: Correspondance, a.a.O.), S. 540 gibt im November-Dezember 1962 „graves difficultés psychologiques“" an. Am 31. Dezember 1962 wird Celan zum ersten Mal in eine psychiatrische Klinik interniert. Vgl. auch James K. Lyon, „Judentum, Antisemitismus, Verfolgungswahn: ,Celans Krise“ 1960-1962“, Celan-Jahrbuch Nr. 3 (1989), S. 175-204.

41. - A. Gellhaus, „Wortlandschaften“, a.a.O., S. 44-51. 
Dabei fällt auf dem mit „13. Feber“ datierten Blatt der Sammelmappe Walliser/Pariser Elegie, deren Aufzeichnungen eine der ersten zum Gedicht In eins führenden Stufen bilden, ein Name auf, den Gellhaus jedoch außer Acht lässt, trotz des korrekten, wenn auch lakonischen Verweises in der Bonner Celan-Ausgabe: Fallik ${ }^{42}$. David Fallik war jener jüdische Maturant im Bukowiner Czernowitz, der 1926 eine Revolte gegen die Willkür von rumänischen, aus dem Altreich in die Maturaprüfungskommission berufenen Professoren anführte, die programmatisch die Kandidaten nicht rumänischer Herkunft, hauptsächlich deutschsprachige Juden, durchfallen ließen; am 10. November 1926, am ersten Tag seines Prozesses, der ihm von den Behörden gemacht wurde, wurde er von einem rumänischen Rechtsextremisten erschossen, der seinerseits von einem Gericht später freigesprochen wurde $^{43}$. Der Fall gewann im kollektiven Gedächtnis der Czernowitzer Judenschaft eine symptomatische Bedeutung, denn damit wurden die Illusionen einer allmählichen Integration in die rumänische Gesellschaft und einer rechtsstaatlichen Garantie gegen die alltägliche antisemitische Bedrohung aufgegeben; die unglüickliche politische Entwicklung, die letztendlich bis zur Entrechtung, Vertreibung und Vernichtung der Bukowiner Juden - darunter auch Celans Eltern - führte, schien ab dieser Schwelle, wo der Mord an einem Juden juristisch ,normalisiert ${ }^{6}$ wurde, unaufhaltsam ${ }^{44}$. Die jüdischen Jugendlichen in Czernowitz sahen dennoch in David Fallik nicht nur ein Opfer, sondern auch ein Symbol eines gerechten Widerstands; das Engagement vieler, Celan inklusive, in den linken legalen und illegalen, mit der systemverweigernden Kommunistischen Partei liierten Organisationen, setzte diesen von Fallik verkörperten Geist des Protests und der aktiven Gegenwehr fort ${ }^{45}$.

Jenseits möglicher (und wohl zulässiger) Erklärungen zum plötzlichen Eintrag des Namens Falliks in die für die Elegie bestimmten Skizzen $^{46}$ kann jedenfalls nicht wundern, dass der verzweifelte

42. - BA, 11, S. $419 \mathrm{f}$.

43. - Vgl. u.a. Mariana Hausleitner, Die Rumänisierung der Bukowina, München, Oldenbourg, 2001, S. 166 f.

44. - Vgl. u.a. Marianne Hirsch, Leo Spitzer, Ghost of Home. The Afterlife of Czernowitz in Jewish Memory, Berkeley/Los Angeles/London, Univ. of California Press, 2010, S. $76 \mathrm{f}$.

45. - Edith Silbermann, Czernowitz - Stadt der Dichter. Geschichte einer jüdischen Familie aus der Bukowina, hrsg. von Amy-Diana Colin, Paderborn, Wilhelm Fink, 2015, S. 93 ff.

46. - Man kann z.B. spekulieren, dass es sich auf dem mit „13. Feber“ datierten Blatt auch um ein Homophonie-Spiel handelte: Fallik = phalic (ein Lexem, das in die erotische Thematik der schon verfassten Versionen der Walliser Elegie hineinpasste und auch wörtlich verwendet wurde, vgl. BA. 11, S. 395) oder Fallik = [Kata]falk. Der jüdische Name Fallik soll vom deutschen Falk abgeleitet sein, vgl. Heinrich W. 
Celan des Jahresanfangs 1962 sich an die anscheinend tief in seinem Gedächtnis geprägte Figur des Czernowitzer Fallik erinnerte. Das lange Ringen um den Text des Gedichts Eine Gauner- und Ganovenweise hatte gleichzeitig den Themenkomplex "Czernowitz“ bis in dessen definitiven Titel eingeführt; der Titelentwurf aus der Notiz vom 23. 1.1962 in einem Taschenkalender enthält noch keine Czernowitz-Erwähnung, was ohnehin vermuten lässt, dass eine gewisse Koinzidenz zwischen der Fallik-Notiz und der Titel-Ergänzung ([...]von Paul Celan aus/ von Czernowitz bei Sadagora) nicht auszuschließen sei ${ }^{47}$. Auch bis zu diesem Blatt vom „13. Feber“ enthalten die Entwürfe in der Sammelmappe der Elegie mehrere Textstellen, die auf eine steigende Rückbesinnung auf Momente und Bilder der eigenen Vergangenheit im Spiegel des ihm widerlich-fremd gewordenen Milieus der deutschen Öffentlichkeit der 1960er Jahre hinweisen: „Pontisches. Einstmal am Rand / des Tatarendorfs [...]" (Kleine Walliser Elegie) ${ }^{48}$; ,hättest du das in der eigenen /[...]/ Sprache: das / Nah-gefremdete / Judendeutsch[...]“49; ,,[...] Christian / Rakowski [...] Meer / Mit dem Schimmer der Taiga; Mauten. Mauten der Heimat. Holz- / geruch sommers, am Rand einer Kindheit. Es stand - im Buch stand und unter den Buchen - , es / stand / eine Sägemühle im Wald. [...]; Mauten und / Mauten jenseits, Maut- / hausen [...]“ (Walliser Elegie, Version vom 24. Dezember 1961) ${ }^{50}$, ,[...] Oberhalb Kronstadts, auf / den Karpaten- / Kanzeln, im / Mönchsgebäu [...]; [...] Corina. Im / Bild dieser Wunde / hat sie ihr Grab, eine Mittel- / meerwelle spült sie dorthin mit ihrem / ertrunknem Jerusalemstern [...]“" (Walliser Elegie, Version vom 24. Januar 1962) ${ }^{51}$. Die scheinbar nostalgischen Rückprojektionen lassen sich jedoch mit der Wunde tiefer Leiderfahrung verflechten: das Meer und das Gebirge sorgloser jugendlicher Exkursionen und Liebschaften, deren von dem „Jerusalemstern“ getragene Spur einer Hoffnung dem unerbittlichen Tod nicht entkommt, die romantische Sägemühle im Wald, die schicksalhaft mit „Mauthausen“ und den Todeslagern vereint

Guggenheimer, Eva H. Guggenheimer, Jewish Family Names \& their Origins. An Etymological Dictionary, New York, Ktav, 1992, S. 232.

47. - Das Heine-Zitat aus der zweiten Strophe des Gedichtes An Edom gedenkt eigentlich der ironisch-bitteren ersten: „Ein Jahrtausend schon und länger,/ Dulden wir uns brüderlich,/Du, du duldest, dass ich atme,/Dass du rasest, dulde Ich." Nicht anders dürfte der junge Celan in den 1920er und 1930er Jahren des 20. Jahrhunderts seinen Czernowitzer Alltag als Jude erlebt haben: „Ja, was den Antisemitismus in unserer Schule betrifft, da könnte ich ein 300 Seiten starkes Buch darüber schreiben“, schrieb der Vierzehnjährige an seine Tante in Palästina, vgl. I. Chalfen, Paul Celan ..., a.a.O., S. 51.

48. - BA, 11, S. 397.

49. - Ebd., S. 403.

50. - Ebd., S. 405f.

51. - Ebd., S. 413. 
ist $^{52}$, das Bewusstsein der schmerzlichen Entfremdung von der sprachlichen Identität seiner Vorfahren ${ }^{53}$, die ,östliche', vom jungen Celan in Czernowitz erträumte Utopie, an die der Dichter immer noch glaubte ${ }^{54}$, die jedoch den Kommunisten Rakowski ${ }^{55}$ und den Dichter Mandelstam ins sibirische Exil schickte und (wie er es in seinem Motto der Elegie durch den dafür ausgewählten Vers Jewtuschenkos betonte) ${ }^{56}$ den von den Deutschen ermordeten Juden im ukrainischen Babij Jar kein Denkmal gönnen wollte.

Dadurch bedeutete für Celan die Heraufbeschwörung von David Falliks Namen eine ,meridianhafte ${ }^{6}$ Rückkehr zu den Wurzeln: ,angereichert" von all dem Geschehenen und Erlebten 57 soll er geglaubt haben, aus der mit dem Meuchelmord bezahlten Kühnheit des jungen Czernowitzers jene Widerstandskraft schöpfen zu können, die er Monate später in den letzten Vorstufen von Eine Gauner- und Ganovenweise bekundete ${ }^{58}$. Auf dem Blatt vom „13. Feber“ notierte er mit deutlichem Verweis auf den ermordeten Fallik: „Wahr, auf den

52. - Der Sägemühle-Vers weist allerdings nochmals auf die poetische Technik des „,coaguler“ hin: Verbunden werden hier eine Erinnerung an die Schulferien in einem Bukowiner Gebirgsdorf mit dem/einem Sägewerk - vgl. I. Chalfen, Paul Celan ..., a.a.O., S. 65 -, ein Zitat aus Franz Werfels Gedicht Romanze der Schwermut - vgl. Barbara Wiedemanns Kommentar in Paul Celan, Die Gedichte, Kommentierte Gesamtausgabe, hrsg. v. Barbara Wiedemann, Frankfurt a.M., Suhrkamp, 2003, S. 930 - und die Metapher der Mühlen des Todes aus dem eigenen Gedicht Spät und tief vom Band Mohn und Gedächtnis, vgl. GW, 1, S. 35.

53. - Unter dem ,nahen [...] Judendeutsch“ verstand Celan, der sich in einem Brief vom 3. März 1962 an Reinhard Federmann „,ein aschkenasischer Jude“ nannte, die jiddische Sprache. Vgl. Reinhard Federmann, „In Memoriam Paul Celan“, Pestsäule, H. 1 (Sept.1972), S. 18f.

54. - „Mon espoir est à l'Est“, schrieb er an Petre Solomon am 22. März 1962 (vgl. P. Solomon, Dimensiunea românească, a.a.O., S. 219), kurz nachdem eine Titelvariante von Eine Gauner- und Ganovenweise mit der Formel „gesungen von Pawel Lwowitsch Tselan, Russkij poët in partibus nemetskich infidelium“ im Januar-Februar 1962 ergänzt wurde, vgl. TA [Niemandsrose], S. 62. Auch John Felstiner erwähnte Celans „,trotzige Nostalgie“" nach seinem ,jugendlichen Sozialismus" und dessen „Affinität“ zu Russland in jenen Jahren, vgl. John Felstiner, Paul Celan. Eine Biographie, München, C.H. Beck, 1997, S. $242 \mathrm{ff}$.

55. - Christian Rakowski (1873-1941) war Führer der rumänischen linken Sozialdemokraten, ging 1917 nach Russland, wo er eine Zeitlang zu der Parteispitze der Bolschewiki gehörte, bis er in Stalins Ungnade fiel. Er starb in sibirischer Deportation.

56. - BA, XI, S. 404. Das russische Zitat aus dem berühmten Gedicht von Jewgenij Jewtuschenko wird Celan selbst ins Deutsche übersetzen: „In Babyi Yar, da steht kein Gedenkstein“. Vgl. ebd. 427.

57. - Vgl. Celans Ansprache anlässlich der Entgegennahme des Literaturpreises der Freien Hansestadt Bremen, vgl. GW, 3, S. 186.

58. - Vgl. die Version vom 7. XI. 62: „Aber er bäumt sich, der Baum. / Er / steht““. An die definitive Version fügte er hinzu: ,,[...] Er, / auch er / steht gegen / die Pest“. TA [Niemandsrose], S. 41. 
Kata- / falken, ruht, was dein Wort / verlebendigt ${ }^{\text {"59 }}$. Ein paar Wochen später wird Celan diesen Willen, nicht ,aufzugeben', in zwei Briefen nach Rumänien - an Alfred Margul Sperber und an Petre Solomon - ausdrücklich an die in seiner Czernowitzer Jugend eingeprägte und eingeübte Kampfbereitschaft gegen einen geistig ewigen "Faschismus“ anknüpfen: ,[...] ich sei wieder $d a$, wo ich angefangen habe. - Ja, da bin ich wieder, genau da. Mitsamt jenem ,no pasaran', das in dem Gedicht ,Schibboleth" steht [...]"60. Aus diesem Kon-Text ergeben sich die ersten Annäherungen an den symbolischen Rose/Rosa-Namen; die Zeilen auf dem mit „20.2.1962“ datierten Blatt, in denen Axel Gellhaus den Kern jener „Synopse von Revolutionen und Aufständen im Europa der Moderne" identifizierte, die zur Vorstufe des Gedichtes In Eins geworden ist ${ }^{61}$, enthalten allerdings nicht nur eine Variation auf den zum Titel des nächsten Gedichtbandes gewordenen Namen (,der Niemands-, der Jedermanns-/Rose [...]"), sondern auch eine erneute Andeutung an die heimatliche Bukowina: „Freund-mit-dem-Buch-von / -derBuche“62, wobei auf dem Typoskript des Gedichtes vom „24.5.1962“ zwei denkwürdige Verse auf Rumänisch handschriftlich eingeschoben wurden: „,si de pe la voi eram mai sînt / de pe la voi"“63. Die Obsession der Herkunft wird auf die ebenfalls in der Sammelmappe der Walliser/ Pariser Elegie bewahrten Vorstufen des Gedichtes Es ist alles anders übertragen: Hier treffen sich nicht nur die thematischen Linien des, östlichen ${ }^{6}$ Widerstands (Normandie-Njemen) ${ }^{64}$ und des Exils des Dichters (Der Name Ossip), die für Gellhaus in Bezug auf das Gedicht In Eins galten ${ }^{65}$, sondern auch diejenige des eigenen Bukowiner Hintergrunds - da reihen sich die Figur der Mutter mit ihrer Vorliebe für die deutsche Sprache ihrer zukünftigen Mörder, der Name des Flusses Bug, an dessen Ufer sie ermordet wurde (am Bug kein wohin), und die an die

59. - BA, 11, S. 419.

60. - Brief vom 9. März 1962 in P. Solomon (Dimensiunea românească, a.a.O.), S. 266. An Solomon schrieb er einen Tag vorher auf Rumänisch: ,[...] mă aflu, cu meridianul meu [...], exact acolo de unde am pornit (avec, je peux le dire ici, mon vieux cour de communiste)“", vgl. ebd., S. 218 [Deutsch: ,ich befinde mich mit meinem Meridian genau dort, von wo ich weiter ging"].

61. - Vgl. A. Gellhaus, „Wortlandschaften“, a.a.O., S. 48.

62. - BA, 11, S. 423.

63. - Ebd. 429. [Dt. „Ich gehörte gehöre auch zu euch, zu euch.“] Die ImperfektVariante wurde vom Dichter selbst gestrichen. Die Übersetzung in der BA entstellt leider den Sinn des rumänischen Satzes!

64. - Am 23. Juni 1962 teilte Celan seinem einstigen Czernowitzer Freund Erich Einhorn nach Moskau mit, dass in der Normandie, wo er sich gerade befand, der Film „Normandie-Njemen“ von Jean Dréville im Kino lief. Vgl. Paul Celan-Erich Einhorn, Briefe, hrsg. u. komm. von Marina Dmitrieva-Einhorn, Celan-Jahrbuch, Nr. 7 (1997/98), S. 32 .

65. - A. Gellhaus, „Wortlandschaften“, a.a.O., S. 46f. 
Bukowina erinnernde Paraphrase von Heinrich Heines Deutschland, ein Wintermärchen (,,[...] wie heisst es, dein Land / hinter den Bergen, den Jahren? [...]") ${ }^{66}$.

Der Text auf dem schon oben erwähnten Blatt vom „7.11.62“, der die vorletzten, zum Widerstand aufrufenden Verse von Eine Gauner- und Ganovenweise [...] von Paul Celan (aus Czernowitz bei Sadagora) wie auch die namentliche Erwähnung von Rosa Luxemburg koppelt, klärt ohne weiteres Celans dichterische Veranlagung beim Entstehen der frühen Version des Gedichts Coagula auf. Den nicht zu vergessenden Mord an der aus Polen stammenden deutsch-jüdischen Kommunistin, die sich weigerte, sich zu unterwerfen, nimmt Celan als blutende „Wunde" wahr, die er auch als seine eigene empfindet: die offene Wunde der Konfrontation mit einer (deutschen) ,Kultur' der Intoleranz, die die standhafte Andersheit nicht duldete und sie gnadenlos zu beseitigen trachtete. Celan, der dazu noch die radikale Bildlichkeit der Wunde Rosas aus Kafkas Landarzt hineinfließen ließ, erhob diese zum Rang des vereinenden Bandes der Kämpfenden, d.h. der Denkerin und des Dichters, die über die Zeitlosigkeit der Sterne und das trügerische Treibsandbett der Geschichte gemeinsam an dem magischen, im Dunkeln leuchtenden Hörnerlicht teilhaben: Damit meint er vermutlich jenes Licht erhabener Reinheit des strahlenden Moses (Exodus, 34.29) nach der Offenbarung der (göttlichen) Wahrheit: keren - das zu vermittelnde Gegenwort zum misslichen Alltag -, das dem ersten Übersetzer der hebräischen Bibel ins Latein als „Hörner“" erschien ${ }^{67}$. Die Tatsache, dass dieses zum Widerstand kraftspendende Licht von „deiner / - auch meiner - / rumänischen Büffel[n]"68 ausströmt, soll um so mehr den Konnex des orphischen, von einer ursprünglichen Wahrheit inspirierten Dichters zu derjenigen aufhellen, die beim Anblick der gequälten, aus Rumänien erbeuteten Tiere von der arkadischen Freiheit einer

66. - Die Andeutung auf Heines Deutschland, ein Wintermärchen sollte das schwierige Erbe der illusionierten „deutsch-jüdischen Symbiose“ um so mehr hervorheben, vgl. BA, 11, S. 431 (5. Juni 1962).

67. - Vgl die Übersetzung von St. Hieronymus, cornuta esset facies sua ex consortio sermonis Dei, vgl. http://rabbiartlevine.com/Home/tabid/2652/ID/840/KiTissa-Moses-Horns-Not-a-Mistranslation.aspx. Vgl. auch Rifat Sonsino, Did Moses Really Have Horns?, New York, URJ Press, 2009. Michelangelos Moses-Statue zeigt den jüdischen Propheten mit den das göttliche Licht symbolisierenden Hörnern.

68. - BA, 11, S. 455. 
"Andersheit“" zu träumen wagte ${ }^{69}$, mit der sich auch Celan in seinem soeben erklärten Ideal des ,karpatisch Fixierten“ identifizierte ${ }^{70}$.

Es steht außer Zweifel, dass Celan eine klare Ahnung vom Inhalt des Briefes von Rosa Luxemburg aus dem Breslauer Gefängnis an Sophie Liebknecht von Mitte Dezember 1917 hatte, als er das Motiv der „,rumänischen Büffel“ in die Coagula-Vorstufe vom 6. Dezember 1962 übernahm. Trotzdem, wie schon gesagt, soll er sich an dem verzeichneten Entstehungsdatum des Gedichts erst vorgenommen haben, Rosa Luxemburgs Briefe aus dem Gefängnis zu lesen ${ }^{71}$. Den sich daraus ergebenden Fragen, die mit der Genese des Gedichtes Coagula zusammenhängen, möchten wir weiterhin nachgehen.

Rosa Luxemburg, die in der Führung der deutschen Sozialdemokratie eine der wenigen war, die konsequent gegen die Beteiligung Deutschlands am Ersten Weltkrieg kämpfte, war ab Februar 1915 inhaftiert und verbrachte die nächsten Jahre, die übrigens sehr wichtig für die Erarbeitung ihres zusammen mit Karl Liebknecht verfassten Projektes einer neuen „,internationalistischen“ Linksbewegung - des künftigen „Spartakusbundes“ - waren, in verschiedenen Gefängnissen, aus denen sie erst am 8. November 1918, am Beginn der Revolution in Berlin, frei $\mathrm{kam}^{72}$. Der Bericht von der Episode mit den „rumänischen Büffeln“, von der sie in ihrem Brief aus Breslau an Liebknechts Ehefrau erzählte, basierte auf ihr - trotz "Sicherungsverwahrung“ - zugekommenen Informationen über Fakten, die historisch belegt sind ${ }^{73}$. Eine erste

69. - „Wie anders schien dort die Sonne, blies der Wind, wie anders waren die schönen Laute der Vögel oder das melodische Rufen der Hirten. Und hier - diese fremde schaurige Stadt, der dumpfe Stall, das ekelerregende, muffige Heu mit faulem Stroh gemischt, die fremden furchtbaren Menschen, und - die Schläge, das Blut, das aus der frischen Wunde rinnt...", vgl. Rosa Luxemburg (Briefe aus dem Gefängnis, a.a.O.), S. 60.

70. - Vgl. den Brief Celans an Alfred Margul Sperber vom 12. September 1962, wo er die "heimatlichen Berge und Buchen“ evoziert, in P. Solomon (Dimensiunea românească, a.a.O.), S. 271. Eine ähnliche Botschaft schickte er an Erich Einhorn am 23. Juni 1962: „Man bleibt zuhause“, vgl. Celan-Einhorn (Briefe, a.a.O.), S. 32.

71. - Vgl. Anm. 35.

72. - Vgl. u.a. Annelies Laschitza, Im Lebensrausch, trotz alledem. Rosa Luxemburg. Eine Biographie, Berlin, Aufbau, 2002; Dietmar Dath, Rosa Luxemburg, Frankfurt a.M., Suhrkamp, 2010.

73. - Es stimmte wohl, dass die schon im Dezember 1916 im Süden Rumäniens eingerichtete deutsche Besatzungsmacht ein drakonisches System von Zwangsabgaben seitens der ruralen Bevölkerung organisierte, das u.a. die Beschlagnahme vom Rindvieh zugunsten der Nahrungsversorgung des Heeres, aber auch für Transportzwecke in den besetzten Gebieten und sogar in Deutschland vorsah. Vgl. u.a. Colecțiunea ordonanțelor pentru populația României în coprinsul administrațiunei militare. Textul românesc [...] de Grigore M. Pherekyde, București, Tipografia Cultura, 1918; Grigore Antipa, 
Buchveröffentlichung, die auch diesen Brief enthält, erschien ein Jahr nach ihrem gewaltsamen Tod in einer Ausgabe, bei der als Herausgeber das Exekutivkomitee der Kommunistischen Jugendinternationale fungierte ${ }^{74}$; es folgte bis heute eine große Menge von Ausgaben der Briefe von Rosa Luxemburg in verschiedenen Formaten und Umfängen.

Den besonderen Nachruhm verdankte dieser Brief aber Karl Kraus, der ihn - auch im Zusammenhang mit der Emotion, die die grausame Bluttat der Freikorps-Soldaten auslöste - in der Wiener Arbeiter-Zeitung, in der Ausgabe von Sonntag, dem 23. Mai 1920 entdeckte ${ }^{75}$. Der Text wurde der Redaktion des österreichischen Organs der Sozialdemokratie wahrscheinlich von einem deutschen, in den KPD-Propagandabetrieb eingeweihten Genossen zugespielt und erschien hier noch vor der Berliner Buchveröffentlichung. Kraus befand sich gerade auf der Reise nach Berlin, wo er nur einige Tage später, am 28. Mai 1920, Rosa Luxemburgs Brief im Rahmen einer seiner öffentlichen Lesungen im Berliner Bechstein-Saal, und danach in Dresden und Prag vorlas ${ }^{76}$. Sein Sinn für Dramatik verstärkte die Wirkung des Textes, dessen literarischen Wert er im Verhältnis zum verhängnisvollen Schicksal seiner Autorin meisterhaft hervorzuheben wusste. In der nächsten FackelAusgabe (546-550, von Juli 1920) wurde das ,einzigartige Dokument von Menschlichkeit und Dichtung“" zusammen mit Kraus' Kommentar veröffentlicht ${ }^{77}$. Eine Überraschung kam aber während des Sommers, als der Fackel-Herausgeber einen anonymen Brief aus Innsbruck als Reaktion auf die Publikation erhielt, in dem er die Diktion jener Ideologie zu erkennen glaubte, deren Schergen Rosa Luxemburg zum Opfer fiel. Seine Antwort kam schon während einer Lesung im Wiener Konzertsaal am 21. Oktober 1920 und sie war von einer vernichtenden Drastik: Der Leserbrief sollte eigenst abgedruckt werden, um ,ein Gruseln vor der unausrottbaren Geistesart deutscher Fortpflanzerinnen, die uns das Leben bis zur todsichern Aussicht auf neue Kriege verhunzen wollen, [...] geschehen zu lassen"78. Ausgerechnet die Polemik gegen derartige geistige Auswüchse stellte für Karl Kraus einen gewichtigen Grund dar,

L'occupation ennemie de la Roumanie et ses conséquences économiques et sociales, Paris, Presses Universitaires de France, 1929; Lisa Mayerhofer, Zwischen Freund und Feind. Deutsche Besatzung in Rumänien 1916-1918, München, Peter Lang, 2010.

74. - Rosa Luxemburg, Briefe aus dem Gefängnis, Berlin, Junge Garde, 1920.

75. - S. 9-10, unter der Rubrik „Feuilleton“.

76. - Vgl. Karl Kraus, Rosa Luxemburg, Büffelhaut und Kreatur. Die Zerstörung der Natur und das Mitleid des Satirikers, hrsg. von Friedrich Pfäfflin, Berlin, Friedenauer Presse, 2009, S. 3.

77. - S. $5 \mathrm{ff}$.

78. - Kraus' messerscharfe Polemik gegen die Innsbrucker Korrespondentin wurde auch in der Fackel, Nr. 554-556 (1920), S. 7-12 veröffentlicht. Vgl. Kraus/ Luxemburg, Büffelhaut und Kreatur, a.a.O., S. 13. 
Rosa Luxemburgs Brief wiederholt in sein Lesungen-Repertoire aufzunehmen - noch am 31. Mai 1928 hat er ihn in Karlsbad vorgelesen ${ }^{79}$.

Es wundert nicht, dass ein Echo dieser Krausschen Begeisterung bis nach Czernowitz, wo Die Fackel in den deutschsprachigen Intellektuellenkreisen immer noch intensiv gelesen wurde ${ }^{80}$, gelangte. Die linke sozialdemokratische Zeitschrift Die Gemeinschaft, die von Albert Maurüber, einem alten Verehrer Kraus', herausgegeben wurde, hat in ihrer Nr. 4 vom 15. Januar 1929 den ganzen Text des Briefes von Rosa Luxemburg von Dezember 1917 veröffentlicht, allerdings ohne eine bestimmte Quelle anzugeben ${ }^{81}$. Celan war damals noch zu jung, um solche Publikationen zu lesen, aber es kann überhaupt nicht ausgeschlossen werden, dass er in seiner Gymnasiastenzeit - besonders als er Kontakte zu kommunistischen Zirkeln aufgenommen hatte - oder sogar während der sowjetischen Besatzung von Czernowitz 19401941 Interesse dafür und Zugang dazu hatte ${ }^{82}$. Jedenfalls kann man mitnichten annehmen, dass er in Czernowitz ein Fackel-Leser war und um so weniger später in seinen Pariser Jahren wurde; Celans Vorbehalte gegenüber Karl Kraus sind übrigens gut bekannt (,un homme douteux, une œuvre douteuse" $)^{83}$.

Dass Celan 1962 bei der Niederschrift des ersten Entwurfs vom Gedicht Coagula den Brief Rosa Luxemburgs mit seinem für ihn damals so wichtigen Verweis auf die „rumänischen Büffel“ kannte, steht andererseits außer Zweifel. Seine Notiz darüber, Rosa Luxemburgs Briefe aus dem Gefängnis lesen zu müssen ${ }^{84}$, besagt nicht, dass er nie eine der Ausgaben in der Hand hatte. Es ist sehr wahrscheinlich,

79. - Ebd., S. 24. Rosa Luxemburgs Brief wurde von Karl Kraus zwischen 1920 und 1928 zehn Mal öffentlich vorgelesen.

80. - Vgl. Amy Colin, ,,Karl Kraus und die Bukowina. Ein unbekanntes Kapitel in der Kraus-Rezeption“, in: Joseph P. Strelka (Hg.), Karl Kraus. Diener der Sprache, Meister des Ethos, Tübingen, Francke, 1990, S. 333-346.

81. - Auf S. 4 unter der Überschrift ,Zum Andenken an die am 15. Januar 1919 ermordeten Führer des Proletariats Karl Liebknecht und Rosa Luxemburg“. Vgl. Andrei Corbea-Hoisie, „Die Czernowitzer ,Gemeinschaft“ (1928-1930). Zur Bestandaufnahme des ,kulturellen Feldes' deutscher Sprache in der Bukowina der Zwischenkriegszeit", Deutsche Vierteljahresschrift für Literaturwissenschaft und Geistesgeschichte, 79. Jg., H. 4 (2005), S. 634-652.

82. - Israel Chalfen war es, der eine Verbindung zwischen dem Interesse Celans für die Figur Rosa Luxemburgs mit der Erinnerung an seine Liebesbeziehung zu der jungen Kommunistin Rosa Leibovici herstellte, die von Jassy nach Czernowitz übersiedelte, nachdem die Nordbukowiner Stadt 1940 von den Sowjets besetzt wurde. Vgl. I. Chalfen, Paul Celan..., a.a.O., S. 144, 150. Seine Behauptungen wirkten so stark nach, dass sie sogar in die Coagula-Kommentare von Olsson (,,Spectral Analysis“, S. 274) oder von Wiedemann (Paul Celan, Die Gedichte, a.a.O., S. 742) eingegangen sind.

83. - Vgl. Celans Brief an seine Frau vom 19.1.1965, in: Paul Celan, Gisèle CelanLestrange, a.a.O., Bd. 1, S. 199; Bd. 2, S. 196.

84. - Vgl. Anm. 35. 
sogar dank dem Krausschen Einsatz für die Autorin, dass das Buch im Vorkriegs-Czernowitz zirkulierte, so dass er es vielleicht in der umfangreichen Bibliothek des linkssozialistischen jüdischen Kulturhauses „Morgenroit" ${ }^{45}$ oder während der gemeinsamen Lektüren sozial-politischer Literatur im Kreis der Freundinnen und Freunde derselben militanten Gesinnung 86 zur Sicht bekam und es mitsamt der Evokation der „rumänischen Büffel“ im Gedächtnis behalten hatte. Dass die Entstehung der ersten Coagula-Version sich zeitlich mit jener außergewöhnlichen Beharrlichkeit deckt, mit der Celan in einer für ihn besonders schwierigen Lebensphase die eigene ,karpatische Fixiertheit" artikulierte, kann letzten Endes auch als Konsequenz dieser Czernowitzer Jugendreminiszenz angesehen werden.

(Kleiner Exkurs) Noch eine letzte Hypothese bliebe zu prüfen. Die von Celan sehr geachtete deutsch-jüdische Philosophin Margarete Susman, die von den Figuren von Rosa Luxemburg und Gustav Landauer, den zwei prominenten jüdischen Opfern der Freikorps-Verbände während der Niederschlagung der kommunistischen Aufstände in Berlin und München im Jahr 1919, sehr eingenommen war, hatte schon sehr früh die Briefe Rosa Luxemburgs aus dem Gefängnis kommentiert ${ }^{87}$ und ist nochmals in einem Aufsatz von 1951 gesondert auf den Brief von Dezember 1917 an Sophie Liebknecht zurückgekommen ${ }^{88}$. Die schweizerische ,religiös-soziale“ Zeitschrift Neue Wege, in der der letztere erschien, gehörte aber sicherlich nicht zu den Lektüren Celans in seinen ersten Jahren in Frankreich. Er besaß zwar ein Exemplar der zweiten Auflage (1948) von Margarete Susmans Das Buch Hiob

85. - Vgl. Hugo Gold (Hg.), Geschichte der Juden in der Bukowina, Bd. 2, Tel Aviv, Olamenu, 1960, S. 142f.

86. - Edith Silbermann nennt ausdrücklich Rosa Luxemburgs Schriften unter jenen, die in derartigen Jugendzirkeln gemeinsam gelesen und debattiert wurden, wobei Celan zu den aktivsten Teilnehmern an diesen Treffen zählte. (Dies hätte übrigens auch der Fall der im Exil-Verlag Querido erschienenen Briefe aus dem Gefängnis Ernst Tollers sein können). Vgl. E. Silbermann, Czernowitz - Stadt der Dichter, a.a.O., S. 95f. Es gab in Czernowitz auch sehr gut bestückte Privatbibliotheken, wie jene des Vaters von Edith Silbermann, Karl Horowitz, zu denen der junge Celan freien Zugang hatte, oder ihres zukünftigen Gatten Jakob Silbermann, vgl. ebd. 86f., 151f. I. Chalfen zitiert seinerseits Zeugen wie Ruth Lackner, Gustav Chomed oder Jakob Silbermann, die behaupten, Celan habe Kafkas Landarzt (mit seiner Rosa-Figur) zum ersten Mal dank Karl Horowitz' Bibliothek gelesen. Vgl. I. Chalfen, Paul Celan..., a.a.O., S. 63, 67f.

87. - Margarete Susman, „Rosa Luxemburgs Briefe“, Der Aufstieg vom 19. Januar 1923.

88. - Margarete Susman, „Rosa Luxemburg“, Neue Wege, Nr. 45, H. 11 (1951), S. 435-440. 
und das Schicksal des jüdischen Volkes ${ }^{89}$; das Exemplar soll er in den 1950er Jahren erworben und intensiv gelesen haben ${ }^{90}$, nur wird darin die Problematik um Rosa Luxemburg überhaupt nicht thematisiert ${ }^{91}$. Deutliche Lektürespuren aus Margarete Susmans Texten über Rosa Luxemburg (und übrigens auch über Gustav Landauer, der ebenso wie Mandelstam zu jenen Intellektuellenfiguren gehörte, mit denen sich Celan gerne zu identifizieren pflegte) ${ }^{92}$ befinden sich erst in dem Exemplar der Ausgabe ihrer gesammelten Aufsätze, das er 1966 - nach dem Tod der Autorin, die er zum ersten Mal am 9. Mai 1963 in Zürich besuchte - vom Herausgeber Manfred Schlösser erhalten hatte ${ }^{93}$. Erst jetzt soll Celan auch den Aufsatz Susmans über Rosa Luxemburg aus dem Jahre 1951 gelesen haben, wo er nicht nur den Ausdruck „rumänische Büffel" durch Anstreichungen hervorhob, sondern auch damit auf das damals schon entstandene Gedichtepaar Solve und Coagula hinweis ${ }^{94}$. Die zahlreichen Lesespuren im Text zeugen davon, dass diese Lektüre ihm wie eine überraschende Offenbarung vorkam: Mit Ausrufezeichen markierte er zum Beispiel eine Wendung, die in Bezug auf Rosa Luxemburgs „Güte“ unmittelbar der alchemischen Formel

89. - Margarete Susman, Das Buch Hiob und das Schicksal des jüdischen Volkes, Zürich, Steinberg, 1948. Der Titel wurde in der Marbacher Paul Celan-Bibliothek mit dem Hinweis registriert, dass er sich nicht im Bestand des Deutschen Literaturarchivs befindet.

90. - Lydia Koelle, „Hoffnungsfunken erjagen. Paul Celan trifft Margarete Susman", in: Hubert Gaisbauer, Bernhard Hain, Erika Schuster (Hg.), Unverloren. Trotz allem. Paul Celan-Symposium Wien 2000, Wien, Mandelbaum Verlag, 2000, S. 85-145, hier 93-102.

91. - Vgl. Elisa Klapheck, Margarete Susman und ihr jüdischer Beitrag zur politischen Philosophie, Berlin, Hentrich \& Hentrich, 2014; Anke Gilleir, Barbara Hahn (Hg.), Grenzgänge zwischen Dichtung, Philosophie und Kulturkritik. Über Margarete Susman, Göttingen, Wallstein Verlag, 2012.

92. - Celan erwähnt Gustav Landauer neben Piotr Kropotkin in seiner MeridianRede als Autoren, mit deren Schriften er aufgewachsen sei. Vgl. GW, 3, S. 190.

93. - Margarete Susman, Vom Geheimnis der Freiheit. Gesammelte Aufsätze 1914-1964, hrsg. v. Manfred Schlösser, Darmstadt/Zürich, Agora, 1966. Ein anderes Buch von Margarete Susman (Die geistige Gestalt Georg Simmels, Tübingen, Mohr, 1959), das Celan seit 1960 besaß, hatte er anscheinend nicht gelesen (vgl. L. Koelle, „Hoffnungsfunken erjagen“, a.a.O., S. 135).

94. - Es handelt sich um eine Unterstreichung und eine Randstreichung auf der Seite 279, vgl. Paul Celan, Bibliothèque philosophique. Catalogue raisonné des annotations, hrsg. v. Alexandra Richter, Patrik Alac u. Bertrand Badiou, Paris, Éditions Rue d'Ulm, 2004, S. 550. Eine andere Randstreichung Celans auf derselben Seite betrifft ein Zitat Susmans aus Rosa Luxemburgs Brief an Sophie Liebknecht von Mitte Dezember 1917: ,„...] ich stand davor und das Tier blickte mich an, mir rannen die Tränen herunter - es waren seine Tränen, man kann um den liebsten Bruder nicht schmerzlicher zucken, als ich in meiner Ohnmacht um dieses stille Leid zuckte", wobei er seine ,Verbrüderung' im Leid mit Rosa Luxemburg im Sinn gehabt haben soll, die er schon 1962 beim Entstehen der ersten Fassung von Coagula ahnte. Ebd. 
solve et coagula zu entsprechen schien ${ }^{95}$. Dass Celans Wissen über Rosa Luxemburgs Brief aus dem Breslauer Gefängnis mitsamt dem in Coagula schon 1962 eingeführten Motiv der „rumänischen Büffel“ von anderswo - am ehesten aus einer im Czernowitz seiner Jugend zu verortenden Quelle - als durch die Vermittlung des Werkes von Margarete Susman stammte, steht jedenfalls fest.

(Nachspiel) Ausgerechnet jenes Gedicht aus dem Jahre 1967, das zum prinzipiellen Streit zwischen Peter Szondi und Hans Georg Gadamer über die Interpretation der Celanschen Lyrik führte ${ }^{96}$, setzte Paul Celans poetische Reflexion über Rosa Luxemburgs Märtyrertod fort. Der Dichter hatte im Dezember eine Einladung angenommen ${ }^{97}$, in der Berliner Akademie der Künste und im von Peter Szondi geleiteten Komparatistik-Institut der Freien Universität Gedichte zu lesen. Man war dabei mit seinem Werk vertraut, so dass er als Nachtlektüre nicht zufällig ein kürzlich erschienenes Buch über die Ermordung von Rosa Luxemburg und Karl Liebknecht erhielt ${ }^{98}$; auf dem Weg zum Gästehaus wurde er auch an dem neu erbauten Appartementhaus, das auf demselben Platz stand und denselben Namen „Eden“ wie jenes Hotel trug, in dem man das Verbrechen gegen die beiden Kommunistenführer bewerkstelligte, vorbeigefahren und dann entlang des Landwehrkanals, in dem man einst die Leiche Rosa Luxemburgs fand 99 . Das Gedicht, das Celans gewaltiges Empfinden der Nähe am Tatort aufgenommen

95. - „Einfach und schlicht gut sein, das löst und bindet alles“, vgl. M. Susman, Das Buch Hiob..., a.a.O., S. 274. Vgl. Bibliothèque philosophique, a.a.O., S. 549.

96. - Vgl. Anm. 14.

97. - Zwei Tage nachdem Celan am 21. November 1967 einen Brief an Peter Szondi mit Details über den künftigen Berlin-Aufenthalt schickte (vgl. Peter Szondi, Briefe, hrsg. v. Christoph König u. Thomas Sparr, Frankfurt a.M., Suhrkamp, 1994, S. 244 f.), schrieb Celan jenen Brief an Petre Solomon, der im Gedicht Coagula die Referenz an Rosa Luxemburg signalisierte (Dimensiunea românească, a.a.O.).

98. - Peter Szondi erinnerte sich, Celan die damals neu erschienene Dokumentation zum Mordfall Liebknecht-Luxemburg von Elisabeth Hannover-Drück und Heinrich Hannover (Der Mord an Rosa Luxemburg..., a.a.O.) geschenkt zu haben. Vgl. P. Szondi, „Eden“, a.a.O., S. 118.

99. - „Der Hohn, den die Beibehaltung des Namens für das Luxusappartementhouse auf das Gedenken der beiden Ermordeten darstellt, war Thema unseres Gesprächs im Auto“, ebd. Vgl. auch Marlies Janz, ,„...noch nichts Interkurrierendes‘. Paul Celan in Berlin im Dezember 1967“, Celan-Jahrbuch, Nr. 8 (2001/02), S. 335-346. Die nächtliche Fahrt führte dann weiter bis zur Ruine des Anhalther Bahnhofs, dort wo der junge, sich im Zug nach Paris befindende Celan im November 1938 den „Rauch von morgen“ der brennenden Synagogen sah - wie es in seinem Gedicht La Contrescarpe vom Niemandsrose-Band steht (GW, 1, S. 282 ff.). 
hatte ${ }^{100}$, entstand in der Nacht vom 22. auf den 23. Dezember 1967: „Der Mann ward zum Sieb, die Frau / musste schwimmen, die Sau, / für sich, für keinen, für jeden - / Der Landwehrkanal wird nicht rauschen [...]"101. Gewidmet dem in Ost-Berlin lebenden Peter Huchel, sollte es zunächst Wintergedicht betitelt werden ${ }^{102}$, denn es war tatsächlich Winter in Berlin in jenen Dezember-Tagen: ,il a fait froid et j'ai vu, pour la première fois depuis vingt ou vingt-deux ans, un hiver fait de neige et de neige", schrieb er am 23. Dezember an seine Frau Gisèle103. Die Erinnerung an Rumänien, wo er sich „vor zwanzig oder zweiundzwanzig Jahren“ befand - und vielleicht auch an die „rumänischen Büffel" -, erwachte damit wieder.

Einer der ersten, der eine Niederschrift des Gedichtes mit dem veränderten (und definitiven) Titel $\mathrm{Du}$ liegst durch eine von Tegel kurz vor dem Flug nach Paris am 29. Dezember 1967 geschickte Briefsendung erhalten hat, war Klaus Reichert, Celans neuer Lektor im Suhrkamp Verlag in Frankfurt am Main ${ }^{104}$. Klaus Reichert bedankte sich, indem er Celan „am 70. Geburtstag Brechts“ (d.h. am 14. Februar 1968) ein Buch schenkte: Es handelte sich ausgerechnet um Rosa Luxemburgs Briefe aus dem Gefängnis in einer 1932 nachgedruckten Auflage und erweiterten Version der ersten Ausgabe (Nr. 10 der Reihe „Internationale Jugendbibliothek") von $1920^{105}$.

100. - Den Kern seiner Interpretation des Gedichtes Du liegst, die der unbeendete Aufsatz „Eden“ (Celan-Studien, a.a.O.) enthalten sollte, hatte Szondi in einer kleinen Notiz festgehalten: „Darüber, das nichts stockt, stockt das Gedicht. Dass nichts stockt, macht das Gedicht stocken“ (ebd., S. 134). Vgl. auch die Interpretation von Hans-Michael Speier in: Ders. (Hg.), Gedichte von Paul Celan. Interpretationen, Stuttgart, Reclam, 2002, S. 175-196.

101. - Schon Szondi bemerkte, dass Celans Verse aus manchen Aussagen der Täter, die er in der erhaltenen Dokumentation fand, ,zitieren“: Liebknechts Leiche war von Kugeln wie ein „Sieb“ durchlöchert, Rosa Luxemburgs Leiche wurde in den Landwehrkanal geworfen. Vgl. P. Szondi, „Eden“, a.a.O., S. 119.

102. - Celan betrachtete dieses Gedicht als eine Art, Antwort' auf Peter Huchels Gedicht Winterpsalm. Es wurde zum ersten Mal in Hommage für Peter Huchel, hrsg. v. Otto F. Best, München, Piper, 1968, S. 16 veröffentlicht. Celan bereitete es für die Publikation im Gedichtband Schneepart vor, der 1971 posthum erschien. Vgl. GW, 3, S. 334.

103. - Correspondance, a.a.O., Bd. 1, S. $596 \mathrm{f}$.

104. - Joachim Seng, ,,Nichts/stockt". Ein Berlin-Gedicht Celans und seine Geschichte“, in https://www.academia.edu/34076106/_Nichts_stockt_Ein_BerlinGedicht_Paul_Celans_und_sein_Geschichte. Überarbeitete Fassung eines Beitrages aus: Anne Bohnenkamp, Waltraud Wiethölter (Hg.), Der Brief - Ereignis und Objekt. Katalog einer Ausstellung im Freien Deutschen Hochstift - Frankfurter GoetheMuseum, Frankfurt a.M., Stroemfeld Verlag, 2008, S. 210-212.

105. - Vgl. Bibliothèque philosophique, a.a.O., S. 502. Das von Klaus Reichert 1964 in Frankfurt am Main erworbene Exemplar befindet sich im Besitz der Paul CelanBibliothek im Deutschen Literaturarchiv Marbach. 